\title{
Identificación y cambios de frecuencia de las arvenses en áreas cañeras de Cuba
}

Identification and frequency changes of weeds in sugarcane crop areas of Cuba

Bárbara Caridad Barreto Pérez ${ }^{1,4 \bowtie}$, Rigoberto Martínez Ramírez ${ }^{1}$, Rafael Zuaznábar Zuaznábar ${ }^{1}$, Maribel González Hidalgo ${ }^{1}$, Ramírez Hernández Teresita. ${ }^{2}$, Dávila Lezama María del Rosario $^{2}$, Ortiz Romero Hilario ${ }^{2}$, Aguirre López Enrique ${ }^{3}$

${ }^{1}$ Instituto de Investigaciones de la Caña de Azúcar (INICA). Carretera CUJAE km. 11⁄2, Boyeros, La Habana, Cuba. Teléfonos: (537) 260 2571, (537) 262 4436-37. ${ }^{3}$ Facultad de ciencias Biológicas y Agropecuarias Universidad Veracruzana. Campus Córdoba-Orizaba.

${ }^{4}$ Facultad de Ciencias Agrícolas. Universidad Veracruzana. Zona Xalapa. México.

${ }^{\boxplus u t o r ~ p a r a ~ c o r r e s p o n d e n c i a: ~ b a r r e t o @ i n i c a . a z c u b a . c u ~}$

Recibido: $19 / 08 / 2016$

Aceptado: 26/11/2016

\section{RESUMEN}

En la zona tropical, donde se encuentra Cuba, las poblaciones de arvenses son generalmente elevadas en los cultivos y si no se establece un conjunto de medidas para su manejo, pueden acarrear pérdidas superiores a $25 \%$ de la producción de las cosechas. La investigación se realizó con el objetivo de identificar las especies de arvenses presentes y los cambios de frecuencia de éstas, en las áreas cultivadas con caña de azúcar de la provincia Artemisa, para sobre esa base, recomendar un manejo integrado para su control. Para la realización del trabajo se utilizó la base de datos de las encuestas de malezas realizadas por el Servicio de Control Integral de Malezas (SERCIM) en las dos Unidades Empresariales de Base (UEB) de la provincia, en el período comprendido desde 2007 hasta 2015. Se informó la presencia de 19 especies, de las cuales dos se clasifican como Muy frecuentes, dos Medianamente frecuentes, dos Poco frecuentes y 13 Accidentales. De ellas, se incrementan tres especies, tres decrecen y 13 se mantienen estables en los campos.

Palabras claves: caña de azúcar, provincia Artemisa, categorías de frecuencia de malezas, manejo integrado de malezas.

\section{ABSTRACT}

In the tropics, where is located Cuba, weed populations are generally high in cropped soils and if a set of measures for its management is not established, can carry more than $25 \%$ of crop production losses. The research was conducted in order to identify the weed species present and its frequency changes in sugarcane cropped areas at Artemisa province, to establish an integrated management control. The database weeds surveys conducted by the 
Integrated Service Weed Control (SERCIM) in two Enterprises Units Base (UEB) of the province, in the period ranging from 2007 to2015. It was informed the presence of 19 species, out of which two are in the category frequent; two fairly common, two uncommon and 13 accidental. Three species are increasingly, three are decreasing and 13 are stable in the field.

Keywords: sugarcane, Artemisa province, frequency categories of weeds, integrated management of weeds.

\section{MATERIALES Y METODOS}

\section{INTRODUCCIÓN}

La presencia de arvenses compitiendo con el cultivo influye decisivamente en el desarrollo de las plantaciones cañeras, su interferencia ocasiona pérdidas en los rendimientos, además de comportarse como hospederas de plagas (León, 1987).

Es necesario conocer el ciclo de vida, modo de reproducción y frecuencia de aparición, entre otras características, de la

diversidad de especies de arvenses diseminadas en las áreas asociadas al cultivo de la caña de azúcar, para la elaboración de estrategias apropiadas y efectivas para su control, y así contrarrestar el aumento considerable de las pérdidas que se producen en el proceso productivo (Cartaya, 1990 y Hernández et al., 2009).

En Cuba Díaz (2003) señaló 32 especies principales que compiten con este cultivo. Varios investigadores consideran necesario actualizar periódicamente los estudios de identificación de arvenses; ya que el uso de determinados métodos de control, producen variaciones en la emergencia y la composición de muchas de las especies de un año a otro, agregándose a ello la introducción de nuevas arvenses en la zona (Barrera, 2010). Teniendo en cuenta lo anterior se planteó como objetivo la identificación de las principales malezas presentes y evaluación de su frecuencia de aparición en áreas cañeras de la provincia de Artemisa en la etapa comprendida desde 2007 hasta 2015 con la finalidad de determinar las especies más importantes y la planificación de estrategias adecuadas para su control.
Para la ejecución de la investigación se tomaron los datos aportados por las encuestas de identificación de malezas realizadas por el Servicio de Control Integral de Malezas (SERCIM) desde el año 2007 hasta el 2015 en las Unidades Empresariales de Base (Harlem y 30 de Noviembre), ambas pertenecientes a la provincia de Artemisa. La identificación se realizó por el método visual según Bernal y Arzóla (2010). Los campos se recorrieron por sus diagonales y se utilizaron manuales de identificación de malezas. La distribución de las especies en el campo se determinó a través de su frecuencia relativa, la que se calculó mediante la fórmula: $\mathrm{F}(\mathrm{x})=(\mathrm{A} / \mathrm{B}) \times 100$; dónde $\mathrm{A}$ es el número de muestras en que concurre la especie y $\mathrm{B}$ es el número total de muestras tomadas. De acuerdo con el valor de frecuencia establecido por Díaz (2003) las especies se clasificaron en las categorías de: accidental $(<25 \%)$; poco frecuente (desde $\geq 25$ hasta $\leq 49 \%$ ); medianamente frecuente (desde $\geq 50$ hasta $\leq 74 \%$ ) y muy frecuente (desde $\geq 75$ hasta $\leq 100 \%)$.También se realizó la clasificación botánica de las mismas en Clases y familias. La evolución de las especies botánicas se clasificó en: crecen, decrecen y estables.

\section{RESULTADOS Y DISCUSIÓN}

Las encuestas realizadas para identificar las arvenses asociadas al cultivo de la caña de azúcar mostraron un total de 19 especies distribuidas en 7 familias y 16 géneros. De las familias identificadas dos 
pertenecen a la clase Liliatae (63.2\%) y cinco a la Magnoliatae (36.8 \%). La familia más diversa fue la Poaceae que agrupó 9 géneros y 11 especies, $(57.89 \%)$ de las encontradas (figura 1). Estos resultados coinciden con los informados en otros países, al citar la familia Poaceae como la más importante que aportó la mayor cantidad de especies en varios sistemas agrícolas (Hernández y Díaz, 1999; Hernández et al, 2009).

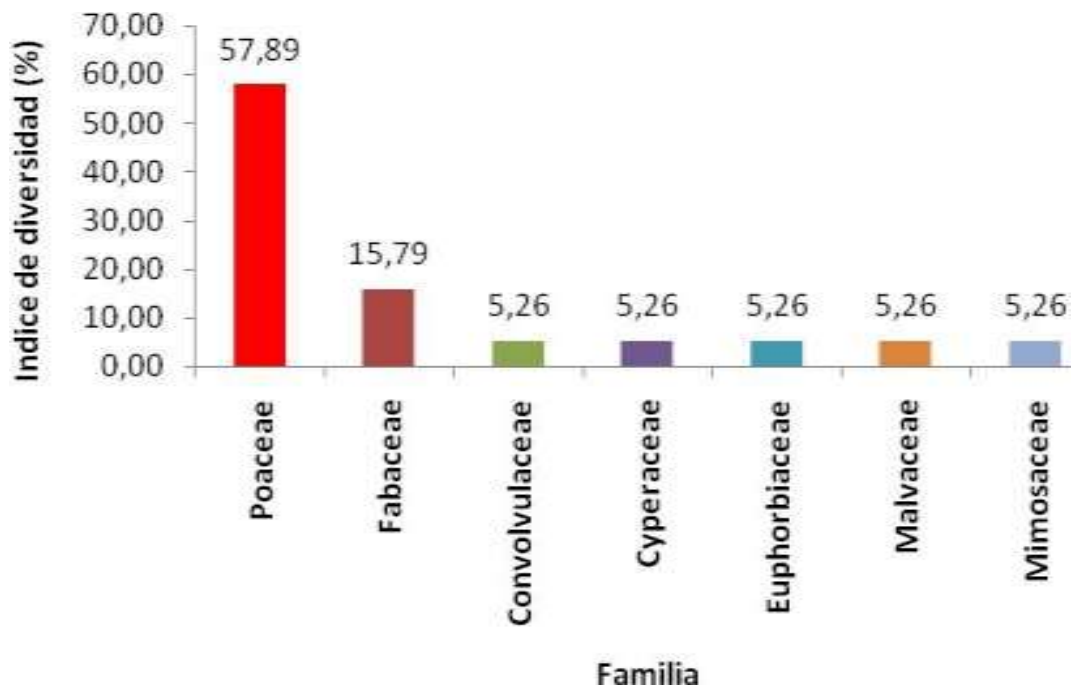

Figura 1. Índice de diversidad de las familias en el año 2015.

De las 19 especies identificadas en las áreas encuestadas, seis son consideradas por el SERCIM como arvenses problema (figura 2), donde el Don Carlos y la Yerba de Guinea ocupan los mayores valores de frecuencia de aparición. Son consideradas especies problemas por el modo de reproducción que presentan, lo que hace más difícil su control.

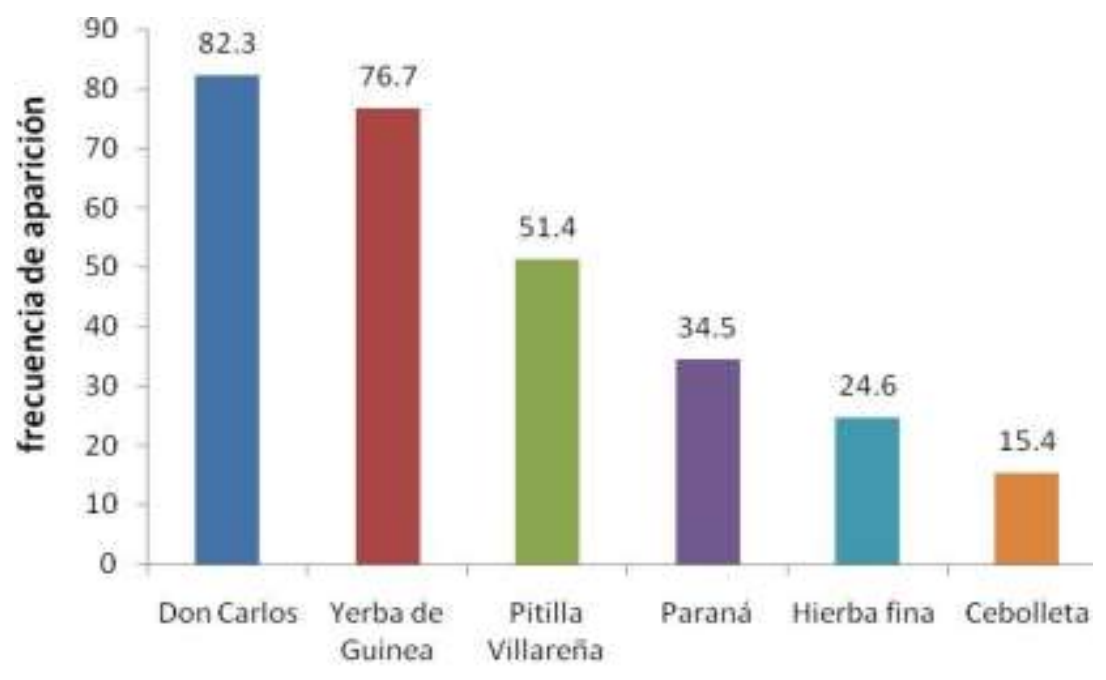

\section{Arvenses problema}

Figura 2. Arvenses problema“, presentes en la empresa azucarera Artemisa en el año 2015. 
Se clasificaron dos malezas muy frecuentes, dos medianamente frecuentes, dos

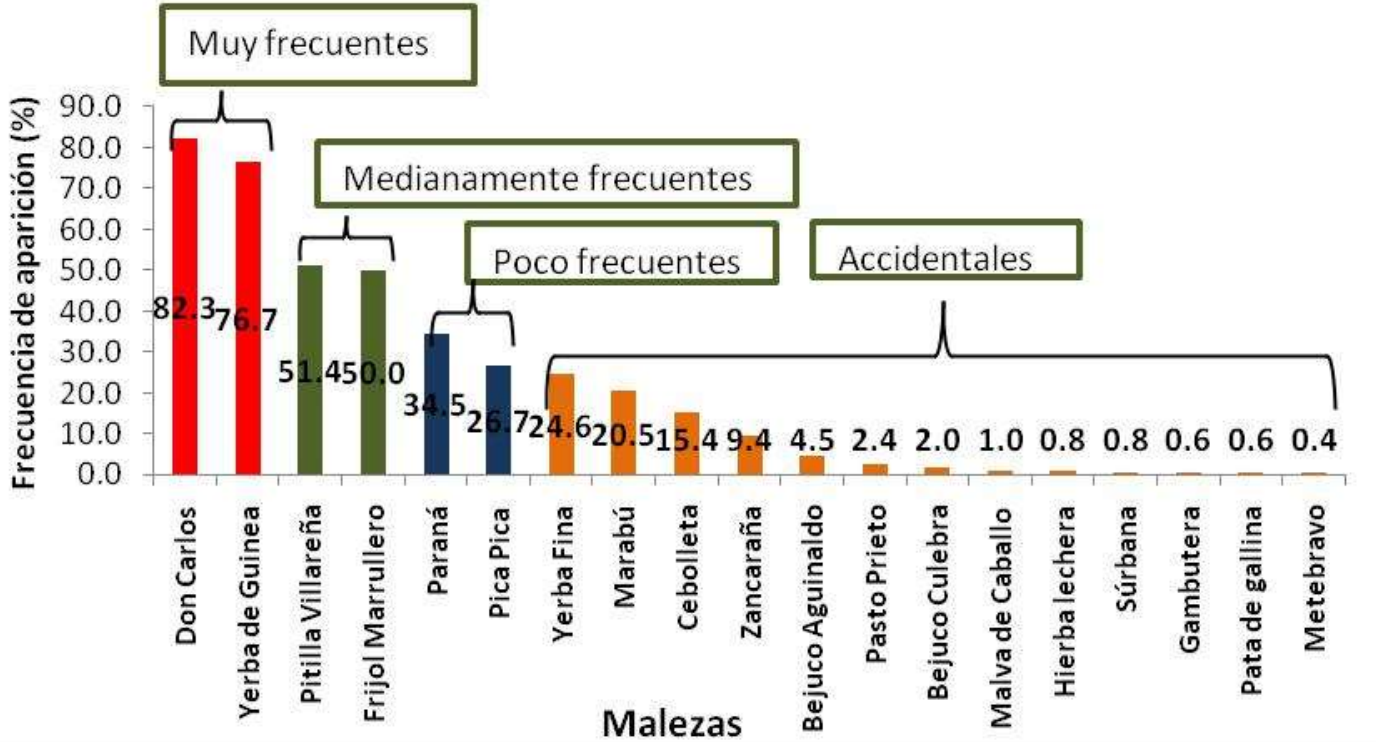

Figura 3. Clasificación de las arvenses identificadas por categorías de frecuencia en la empresa azucarera Artemisa en el año 2015.

El Don Carlos (Sorghum halepense) fue la arvense que mostró el mayor valor de frecuencia $(82.3 \%)$, seguido de la Yerba de Guinea (Panicum maximum) $(76.7 \%)$ en la categoría de muy frecuentes. La presencia de estas arvenses en las áreas cañeras constituyen un serio peligro fitosanitario por el gran riesgo que representan en la transmisión de varias enfermedades (Chinea et al., 2009). Estos valores elevados de frecuencia se deben fundamentalmente en el caso del Don Carlos a ineficiencias en la preparación de suelos, la no observancia del tiempo entre las labores y el uso de implementos inadecuados (Chinea et al., 2007).

Dentro de la categoría medianamente frecuentes se encontraron la Pitilla Villareña (Dichanthium annulatum) y Frijol Marrullero (Vigna vexillata). La forma más efectiva para el control de la primera especie, es la preparación de suelos en seco, con el correspondiente espaciamiento entre labores, combinándolo con el control químico (INICA, 2007). Esta arvense es hoy una de las más comunes asociadas al cultivo de la caña de azúcar, de acuerdo con lo informado por
(Labrada y Parker, 2000; Monroig, 2010., García, 2013 y Barrera et al, 2014).

Como poco frecuentes se clasificaron el Paraná (Brachiaria mutica) y Pica Pica (Mucuna pruriens). Esta última crea dificultades en la cosecha por la obstrucción a las cosechadoras y por su poder urticante, al personal involucrado en la misma (Monroig, 2010) lo que incrementa los costos de cosecha debido a pagos adicionales en áreas de gran infestación.

En el grupo de las accidentales, 8 malezas sobrepasan el 1\%, siendo 6 de ellas las que presentan los más altos valores, la Yerba Fina (Cynodon dactylon) $24.6 \%$, Marabú (Dichrostachys cinérea) 20.5\%,

Cebolleta (Cyperus rotundus) 15.4\%, Zancaraña (Rottboellia cochinchinensi) 9.4\%, Bejuco Aguinaldo (Ipomoea nil) $4.5 \%$ y Pasto Prieto (Brachiaria subquadriparia) $2.4 \%$.

La yerba fina en cultivos de plantación forma manchones que llegan a cubrir superficies considerables, lo que elimina a varias plantas de cultivo, entre ellas la caña de 
azúcar” (León, 1987). El Marabú (Dichrostachys cinérea) cuando no es controlado correctamente puede sobrepasar los valores medios nacionales lo que conllevan a pagos adicionales que incrementan los costos de corte. La cebolleta es agresiva en la caña planta, ya que en ocasiones llega a cubrir toda el área sembrada e inhibe su germinación por su poder alelopático (INICA, 2007). La zancaraña por su característica competitiva y alelopática ocasiona pérdidas de hasta $80 \%$ de las cosechas (Monroig, 2010) por lo que se menciona entre las 18 malezas más problemáticas del mundo. Los bejucos crean dificultades en la cosecha por la obstrucción

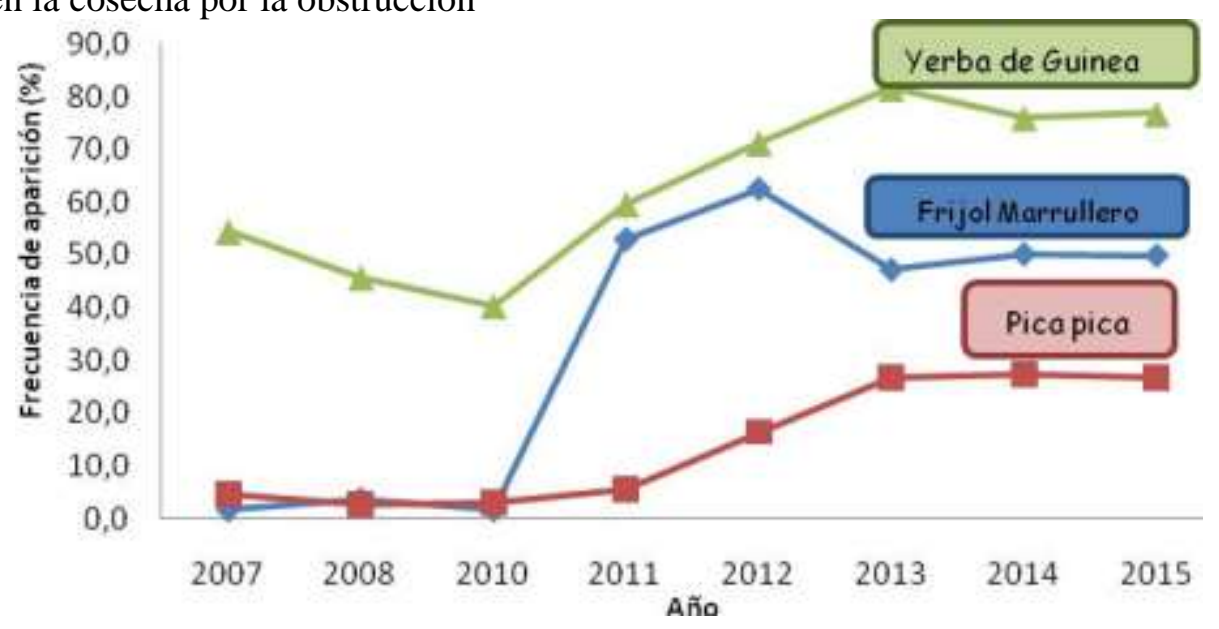

Figura 4. Comportamiento de las Malezas que crecen.

La yerba de guinea (Panicum máximum) fue la arvense que presento el incremento más notable con $22.3 \%$, lo que la clasifica al final del período analizado en una categoría superior a la que mostró a inicios del mismo.

La Pica Pica (Mucuna pruriens) mostró un incremento de $22 \%$, lo cual se debe según Bernal y Arzola (2010) a que el método de control químico que debe realizarse de forma aérea, ha sido poco utilizado en los últimos años por problemas económicos, además de ello, el incumplimiento en el control preventivo, como limpieza de equipos de cosecha, aperos de labranza y restricción de

movimiento desde áreas infestadas (Monroig, 2010). que provocan en las máquinas cosechadoras (Soares 2004).

La comparación de los valores de frecuencia de las arvenses del año 2015 respecto a los del año 2007 muestra que tres especies crecen, Yerba de guinea (Panicum máximum, Frijol Marrullero, (Vigna vexillata) y Pica Pica, (Mucuna pruriens), (figura 3),

Tres decrecen, Paraná 31.0 (Brachiaria mutica), Zancaña $24.9 \quad \% \quad$ (Rottboellia cochinchinensis) y Mete Bravo $5.2 \%$ (Echinochloa colona) y el resto se comportan estables. 
sino que también evita la aparición de otras, al impedir la germinación de sus semillas como consecuencia de su desecamiento por la exposición al sol.

Distancia de plantación: En los campos que se cosechan manual se pueden establecer distancias entre surco cerca de 1 $\mathrm{m}$ lo cual disminuye la infestación de arvenses y el costo de su control. En los campos de cosecha mecanizada se debe establecer la base ancha $0.40-1.40$.

Cultivar: Plantar cultivares de cierre temprano que son más competitivos y reducen $25 \%$ las labores de desyerbe. En la medida que las variedades tengan crecimiento más rápido, el limbo de la hoja sea más ancho, las hojas se curven hacia la horizontalidad, el cierre del campo será más rápido y el autosombreo actuará como regulador del enyerbamiento.

Fertilización: Realizarse en áreas libres de arvenses. El fertilizante se debe aplicar siempre sin presencia de malezas en el cultivo. Cuando la fertilización se realiza a la dosis óptima, pero no se lleva a cabo un adecuado control de las malezas, los rendimientos que se obtienen son inferiores a cuando se ejecuta un buen programa de manejo de las malezas, aunque no se aplique ningún fertilizante

Labores de cultivo: Realizar de 3 a 4 labores. En el entresurco realizarlas con tractores o con tracción animal y en el surco con herbicidas o manualmente.

Control Químico: Realizarlo de forma oportuna, en los estadios más jóvenes de las arvenses y adecuadas condiciones de humedad y mullido del suelo. Aplicar las dosis requeridas para cada situación arvense-cultivo suelo.

Cubierta de residuos: Realizarla en áreas donde el suelo tenga buen drenaje interno y rendimientos agrícolas mayores de $70 \mathrm{t} \mathrm{ha}^{-1}$. En cañas de soca o retoño las cubiertas inalteradas de residuos de cosecha reducen significativamente la infestación de malezas y los costos para su control, al mismo tiempo que conservan la humedad y evitan la erosión del suelo. Disciplina técnica: Realizar el control preventivo como son la restricción del movimiento y limpieza de equipos de cosecha y aperos de labranza desde áreas infestadas lo que evita la diseminación de las malezas de un campo a otro.

\section{CONCLUSIONES}

La identificación de las arvenses realizada muestra que la familia más diversa es la poaceae con 9 géneros y 11 especies.

Las arvenses más frecuentes fueron el Don Carlos (Sorghum halepense) y la Yerba de Guinea (Panicum maximum).

En el periodo comprendido desde 2007 hasta 2015 se incrementa la frecuencia relativa en las especies

Yerba de guinea (Panicum máximum, Frijol Marrullero,(Vigna vexillata) y Pica Pica, (Mucuna pruriens)

\section{RECOMENDACIONES}

Realizar cursos de capacitación que conlleven a un mejor uso de prácticas de control integral de malezas en caña de azúcar.

\section{LITERATURA CITADA}

Barrera, D. F. 2010. La agroindustria de la caña de azúcar en Cuba: retos y amenazas en el nuevo siglo. Revista ATAC, 72 (2): 33-38,.

Barrera, M.; L. Peña; J. Matos; G. Cervera; Midiala Peña; Odalis Barquiet; $H$. Gámez y A. Cobas. 2014.¿Será la encuesta de malezas 
una herramienta para las recomendaciones del Servicio de Control Integral de Malezas? Estudio de caso, de la interacción práctica con los productores. En: Conferencia Técnico-Productiva (XXX: 2014, Junio, 14-15: Palma Soriano). [CDROM]. Conferencia TécnicoProductiva por el XXX aniversario de la Estación Territorial

Bernal, N. y Arzola, N. (2010). Reporte de trabajo de diagnóstico agrícola realizado durante el periodo de mayo 8 a junio 26 del 2010 en el Ingenio Ecudos. S.A, Ecuador. Guayaquil, Ecuador,

Chinea, M. A. 2009: Las enfermedades de la Caña de Azúcar en Cuba durante las últimas cinco décadas. Jornada Científica por el 40 aniversario del INICA. Sección Biológica. Publinica. Cuba. p.79

Cartaya, G., I. Olea, E. Romero y J. Scandaliaris. (1990). Estrategias para el manejo de la grama Bermuda (Cynodon dactylon L. Pers) en caña de azúcar. Rev. Ind. Agrícola (Tucumán), 67(1):11-30.

Chinea, M. A.; Chinea, H. A; Díaz.; J. C. y Hernández, $\mathrm{H}$. $\mathrm{F}$. (2007).Malezasyplantas económicas hospedantes de enfermedades de la caña de azúcar en Cuba. En: Memorias (LX: 2007, Junio, 5-9: Jovellanos). [CD-

ROM]. Memorias del 60 Aniversario de la Estación de Investigaciones de la Caña de

Azúcar "Antonio Mesa", 2007.

ISSN 1028- 6527.

Díaz, J.C. 2003. PC Malezas. Programa Automatizado para el Servicio de Control Integral de Malezas (SERCIM). Rev. ATAC, 65 (3): 8,

García, I. (2013). Evolución de la frecuencia de aparición de las principales malezas que afectan la Caña de Azúcar en Villa Clara.
Informe de la Reunión Nacional de Control Integral de Malezas, Caibarién, enero 2013.

Hernández, D. y Díaz, J.C. (1999). Población de caña y competencia de malezas: efectos sobre el rendimiento agrícola y el contenido de azúcar. Rev.ATAC, 60 (2): p.11-15,

Hernández, H.; Santana, O.; Díaz, J. C.; Chinea, A.; González., Y. (2009) Prueba de extractos de plantas alelopáticas para el control de malezas. Memorias del 60 Aniv. EPICA Matanzas. CDROM ISSN 1028- 6527, Matanzas, Cuba., 7 pp.

INICA (2007). (Instituto Nacional de Investigaciones de la Caña de

Azúcar).Conceptualización

Territorial del Servicio Especializado de Control Integral de Malezas en la Fase de Inversión para el Polígono“Anzoátegui",

Estado Cojedes, Venezuela. InformeAnual. Cojedes, Venezuela, $86 \mathrm{p}$.

Labrada, R. y Parker. C. (2000). El control de malezas en el contexto del manejo integrado de plagas. Organización de las Naciones Unidas parala Agricultura y la

alimentación. Estudio FAO Producción y Protección Vegetal, Roma, Italia., $120 \mathrm{pp}$.

León, D. (1987). Información básica sobre la competencia entre las malezas y los cultivos. UDO. Curso de mejoramiento en protección vegetal. p. 36-43.

Monroig, M.F. (2010). Control integrado de malezas en el cafetal.

En sitio web: http://academic. uprm.edu/mmonroig/id64.htm

Consultado el 2 dediciembre de 2010. 
Soares, M. (2004) Efeito da palha da canade-açúcar y do tamanho de tubérculos no desenvolvimento datiririca (Cyperus rotundus L.). Tesis presentada enopción al Grado

Científico de Doctoral en CienciasAgrícolas. Escola Superior de Agricultura "Luiz de Queiróz", Universidad 
Copyright (c) 2016 Bárbara Cari dad Barr eto Pérez, Rigoberto Martinez Ramirez, Rafael Zuaznábar Zuaznábar, Maribel G onzález Hidalgo, Teresita Ramirez Hemández, Maria del Rosario Dávila Lezam a, Hilario Ortiz Romero y Enrique Aguirre López

\title{
(c) $\underset{\mathrm{BY}}{(\mathrm{i})}$
}

Este tex to está protegido por una licencia licencia Creative Commons 4.0.

\begin{abstract}
Usted es libre para Compartir —copiar y redistribuir el material en cualquier medio o form ato- y Adaptar el documento —remezclar, transformar y crear a partir del material- para cualquier propósito, , incluso para fines comerciales, siempre que cumpla la condición de:

Atribución: Usted debe dar crédito a la obra original de manera adecuada, proporcionar un enlace a la licencia, e in dicar si se han realizado cam bios. Puede hacerlo en cualquier form a razonable, pero no de form a tal que sugiera que tiene el apoyo del licenciante o lo recibe por el uso que hace de la obra.
\end{abstract}

Resumendelicencia - Textocompletodelalicencia 